\title{
A Comparative Study of Mulligan Traction Straight Leg Raising and Dynamic Soft Tissue Mobilization to Increase Hamstringss Flexibility
}

\author{
Basant Kumar Singh, MPT ${ }^{1}$, Mrs. Monalisa Pattnaik, MPT, Asst. Prof. (PT) ${ }^{2}$, \\ Dr. Patitapaban Mohanty, Ph.D., Assoc. Prof. (PT) ${ }^{3}$ \\ Swami Vivekanand National Institute of Rehabilitation Training and Research, Olatpur, Bairoi, Cuttack, India
}

\begin{abstract}
Objective: To compare the effect of dynamic soft tissue mobilisation and Mulligan traction straight leg raise in increasing hamstrings flexibility by active knee extension range of motion and passive SLR.

Research design: Pre test and post test structured, comparative study design.

Participants: Sixty healthy asymptomatic subjects were taken aged 17-30 years from SVNIRTAR, Olatpur Cuttack, Odisha.

Outcome measures: Active knee extension and passive SLR.

Results and conclusion: Mulligan's traction straight leg raise and Dynamic Soft Tissue Mobilization improved active range of knee and passive straight leg raise range of motion in normal individuals. The improvement of control group was not clinically significant.
\end{abstract}

\section{Introduction}

Muscle flexibility plays an important role in prevention of injuries. Flexibility is a key component for injury prevention and rehabilitation. Flexibility does not exist as general characteristics, but is rather specific to a particular joint action. The specificity of flexibility is another example of how the body will adopt to the stresses placed on it. Flexibility is an indisputable component of fitness, defined as the ability to move a joint through a normal range of motion without producing stress to the musculotendinous unit. [1] Much has been written on the importance of flexibility in normal muscle function and prevention of injury. A sedentary life style often results in diminished flexibility. [2] The advantages of having flexibility include increase in ROM, improve joint function, enhance muscle performances, prevent muscle injury and decreases post exercise soreness. Flexibility increases body awareness, better posture and enhances performance of skill movements. [3] Mainly hamstrings flexibility may prevent acute and chronic musculoskeletal injuries, low backache problems, postural deviations, gait limitations and risk of fall. [4] Good muscle flexibility will allow muscle tissue to accommodate to imposed stress more easily and allow efficient and effective movements. [5]

With age, muscles go through a shortening process due to lack of physical activity and loss in elasticity in the connective tissue surrounding the muscle. [6] Factors including arm and leg length, age span, height or weight do not significantly affect ROM. [7] With age, physiological changes that are said to occur includes loss of strength of elasticity in the soft tissue matrixes, diminished capillary blood supply, reduced amount of mesenchymal cells i.e. the stem cells, muscle atrophy and reduced capacity for healing. [8] According to the review of literature that implies that the connective tissue compliance appears to be a major factor in musculoskeletal flexibility. Hamstrings is one of the important muscles during walking. Hamstrings flexibility is always given a greater concern while looking for athlete's overall physical fitness as hamstrings injuries are common and have a significant impact on the performance of an athlete. [9]

Human movements are not possible without a certain amount of the fitness component, commonly called flexibility. To date hamstrings muscle tightness is present in almost all the population of the world which in turn affects the flexibility. Loss of flexibility is defined as a decrease in the ability of a muscle to deform. [26] The tight hamstrings muscles is defined as the inability to extend the knee to less than 20 degree of knee flexion. [8] Several conditions commonly seen by physiotherapist have been linked to hamstrings muscle tightness. [13] A sedentary lifestyle often results in diminished flexibility. [2]

The ability of an individual to move smoothly depends on his flexibility, an attribute that enhances both safety and optimal physical activities. The hamstrings are example of muscle groups that have a tendency to shorten [10]. A tight hamstrings causes increased patellofemoral compressive force, which may eventually lead to patellofemoral syndrome. Flexibility can be enhanced by simple, non-surgical procedures like stretching the shortened muscles. [11] Hamstrings tightness is a common condition found in both symptomatic and asymptomatic subjects. Stretching provides many physical benefits including improved flexibility, improved 
muscle or athletic performance, improved running economy or decreased energy expenditure at a given speed, injury prevention, promotion of healing and prevention of muscle soreness.

Hamstrings muscle tightness is present in almost all population of the world. [12] Several conditions commonly seen by physiotherapist have been linked to hamstrings muscle tightness. [13] Hamstrings muscle tightness with respect to stretching has become one of the most researched topics in the field of orthopaedics and sports medicine today. Poor hamstrings flexibility is often associated with injuries to the lower back and lower extremities. [14] The effects of stretching on flexibility have been well documented in the literature. [14] Stretching has been promoted for years as an integral part of fitness program to decrease the risk of injury. [18] Although evidence to supports the effectiveness of stretching is limited, stretching appears to be in widespread use.

Various stretching techniques and warm up procedures are often suggested prior to sports or physical exercises that are believed to have beneficial effects over flexibility and increase in joint range of motion. [1516] Stretching is important for reducing injury and improving performance in sports and for overall physical fitness. Athletes are often given stretching protocols to improve their flexibility. Several stretching techniques are used to increase joint range of motion (ROM).[17] Stretching has been promoted for years as an integral part of fitness program to decrease the risk of injury.[18] Stretching in a general term used to describe any therapeutic manoeuvre designed to lengthen pathologically shortened soft tissue structures and thereby to increase ROM. [19] Stretching is important because it contributes to various physical benefits including running economy, injury prevention, promotion of healing etc. [20] The other benefits of stretching are namely increase functional range of motion [21], reduction of low back pain and injury [22], reduction of incidence of severity of injury [23], improvement in posture, muscle symmetry and promotion of mental relaxation. A variety of stretching activities has been presented in the literature in order to regain or maintain muscle flexibility and avoid a decrease in range of motion that can impair functional activities in an individual. [5] A study by Hartig. $\boldsymbol{D} . \boldsymbol{E}$ found that a regular hamstrings stretching help to significantly decrease the number of overuse injury that occur with physical training. [24]

D Hopper developed dynamic deep muscle tissue model (DDMT) to treat athletes with muscle tightness and associated soft tissue problems. This DDMT model consisted of a series of progressions from traditional to dynamic techniques which concentrated on one specific area of muscle tightness. It is hypothesized that incorporating active contractions into a massage protocol may increase muscle perfusion and decrease muscle stiffness. Clinical experience suggests that the DDMT model is an efficient, pain free intervention that appears to have an immediate effect on improving hamstrings flexibility. [25]

The purpose of the study is to compare the effect of dynamic soft tissue mobilisation and Mulligan traction straight leg raise in increasing hamstrings flexibility by active knee extension range of motion and passive SLR.

\section{Methodology}

Population: Students with hamstrings tightness from Department of Physiotherapy, Occupational therapy and Prosthetic and Orthotics, SVNIRTAR, Olatpur, Cuttack, Odisha.

Sample Design: The sampling design used for the study was purposive sampling.

Sample Size: Sixty healthy asymptomatic college male students aged 17-30 years from SVNIRTAR, Olatpur, Cuttack from department of physiotherapy, department of occupational therapy, and department of P\&O fulfilling the inclusion criteria were taken into the study and assigned into three groups; group A, group $B$ and group $C$ with 20 subjects in each group.

Study Design: A three groups, Pre test and post test structured, comparative study design.

Duration of the Study: The study was conducted over duration of 12 months.

Inclusion Criteria: Asymptomatic subjects aged 17-30 years, males, right side, 20-40 degrees active knee extension loss with hip in 90 degrees flexion, stretch end feel at the end range, subjects with passive straight leg raise (SLR) of between $40^{\circ}$ and $70^{\circ}$.

Exclusion Criteria: Females, fractures of the hip and knee, dislocations of the lower limb, hamstrings injuries, hyper mobility of the lower limb joint, muscle imbalances of the lower limb, nerve lesions of the lower limb, subjects have low back pain in the last 2 months, straight leg, hip flexion range of motion was greater than $100^{\circ}$, metal pins, plates, or screws in the femur, neurological abnormalities, any pathologies of lower back, hip, thigh and knee.

\section{Procedure}

Sixty asymptomatic healthy male subjects who fulfilled the inclusion and exclusion criteria were randomly selected and written consent was taken. They were then further divided into three groups (20 subjects in each group) and underwent baseline assessment. Experimental group A with mean age year $(23.7 \pm 3.01)$ 
received Mulligan traction straight leg raise. Experimental group B with mean age year $(22.4 \pm 1.83)$ received dynamic soft tissue mobilization, and control group $C$ with mean age year $(20.5 \pm 0.35)$ was control group intervention. The procedure of the intervention and the possible risk involved was explained to each subject. No one dropped out of the study.

\section{Group A- Mulligan Traction SLR}

Position of subject: Supine lying on plinth

Position of Therapist: Standing at lower end of the table

Procedure: This technique involved sustained traction applied to the limb with the knee extended. The subject was in supine lying on a very low bed or on the floor and therapist stood facing patient's affected side. Subject actively did the SLR. Therapist grasped subject's lower leg proximal to the ankle joint and raised it off the bed to a position just short of the painful range. Therapist flexed the subject's knees and held the clasped leg to his chest. Effective longitudinal traction was applied to the leg when the therapist extended the knee. This traction was sustained and straight leg raise was undertaken as far as possible provided with no pain. If pain/severe discomfort noted slight rotation, abduction or adduction of the hip was applied while raising the leg. Pain free SLR with traction was given for three times. Intervention was given on alternative days for 3 weeks.

\section{Group B- Dynamic Soft Tissue Mobilisation}

Subject position: - prone and later came to supine

Therapist position: - By the side of the patient

Procedure- To assess the hamstrings muscle group, the subject remained in the prone position and deep longitudinal strokes were applied to the entire muscle group. Once the specific area of hamstrings muscle tightness/discomfort was located, the remaining intervention was limited to this target area. To execute the dynamic intervention, the subject was made to move into a supine position with the hip and knee flexed to $90^{\circ}$. In this position, all dynamic techniques worked the hamstrings muscle length from three quarter to end ROM. Deep longitudinal strokes were applied in a distal to proximal direction to the area of hamstrings tightness when the leg was passively being moved to the hamstrings lengthened position. Five strokes were applied and 20 seconds of shaking were performed at the completion of this technique. The specific area of hamstrings tightness was reassessed to determine whether the surface area of the site of muscle tightness was reduced. When this reduction occurred, then the next progressive dynamic technique was applied. The same sequence was implemented for the next dynamic technique. During this technique, the subjects required to actively extend their leg, in order to achieve reciprocal inhibition of the hamstrings. In the final technique, the subjects were require to work the hamstrings muscle group eccentrically by creating tension in the therapist's hand as the muscle was elongated to the end ROM. During this movement, the therapist performed five deep distal to proximal longitudinal strokes over the reduced hamstrings area of muscle tightness. Intervention was given on alternative days for 3 weeks.

\section{Group C- Control Group}

The subjects were positioned in prone lying position for a period of 5 minutes every alternative day for 3 weeks

The following dependant variables were measured prior to the beginning of the study and were repeated finally after 3 weeks of intervention.

\section{1) Active Knee Extension Test}

Each subject was positioned in left side lying on examination table for bony landmark identification. The lateral femoral condyle, head of fibula, and lateral malleolus of the right leg were marked to ensure that the same reference points were used for repeated measurements. Once the landmarks were identified, subjects were instructed to lie in supine position. The subject flexed both hips to $90^{\circ}$ and grasps the behind the knees to stabilize hip at $90^{\circ}$. A goniometer was then used to position right knee at $90^{\circ}$. A stationary arm along lateral femur and movable arm aligned with lateral fibula keeping lateral femoral condyle as axis. Subjects actively extend the right knee as much as possible without moving the thigh from vertical position. Active knee ROM was measured by goniometer. This procedure was used for flexibility measures in all the groups.

\section{2) Passive SLR}


Subject was in supine position on right side of the body parallel with the edge of a firm, level table. The trunk and pelvis was place in the anatomical position. One therapist was standing in the testing side of the subject at the foot end. Another therapist was standing in the same side also and measuring the passive SLR with a universal goniometer. The proximal or the fixed arm was placed parallel to the mid-axillary line of the trunk and the movable or distal arm was placed parallel to the longitudinal axis of the femur in line with lateral femoral condyle.

\section{Data Collection}

Measurements were taken prior to the beginning of the study and were repeated finally after the completion of 3 weeks intervention. Subjects were tested on all the dependent variables. Data collected were transcribed onto a data sheet for each subject separately.

\section{Data Analysis}

The dependent variables were analyzed using a 2x2 ANOVA, repeated measures on second factor. There was one between factor (group) with two levels ( Experimental and Control) and one within factor (time) with two levels ( Pre and Post). All pair wise post - hoc comparisons were analyzed using a 0.05 level of significance. Analysis was performed using SPSS versions 16.0 package.

\section{Active knee extension}

\section{Results}

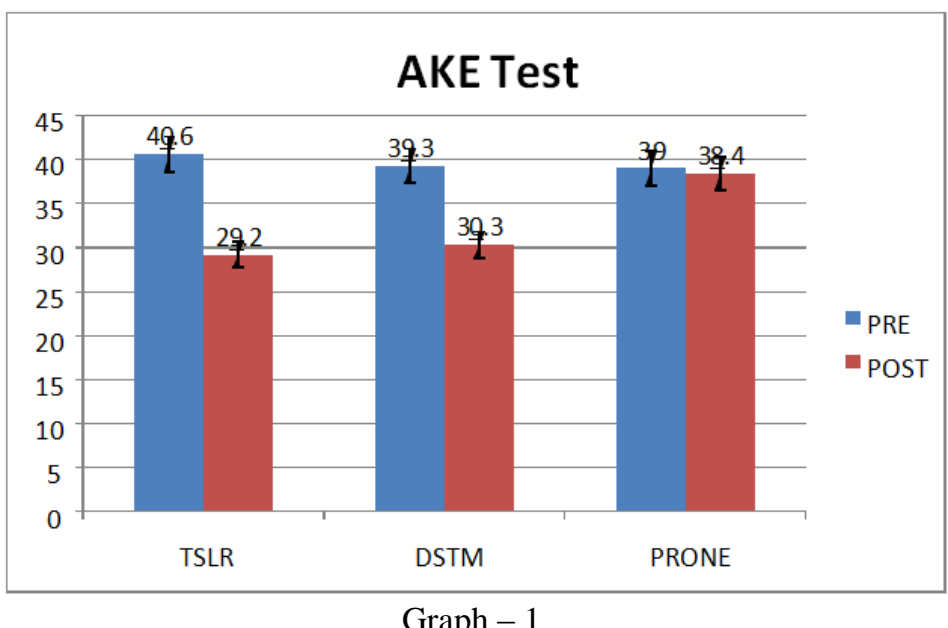

The Graph 1 illustrates that there was improvement in active range of motion in groups receiving mulligan traction straight leg raise (gr-1) and dynamic soft tissue mobilization (gr-2), but not in the control group (gr-3) from pre intervention measurement to post intervention measurement for a period of 3 weeks. Result showing that there was main effects for time i.e., $\mathrm{f}(1,28,0.05)=1.293 \mathrm{E} 3, \mathrm{p}=0.000$ and main effects for groups $\mathrm{f}(1,28,0.05)=2.823, \mathrm{p}=0.0000$ main effects qualified into Time $\times$ Group interaction $\mathrm{f}(1,28,0.05)=$ 282.889. Turkey' post Hoc analysis shows that all the groups improved significantly from pre to post although control group did not have clinically significant changes ( 0.6 degree $)$ at the end of 3 weeks significant difference was seen between all the groups but differences between group- 1 and group- 2 is only 1.1 degree which is not clinically significant. 


\section{Passive Straight leg raise}

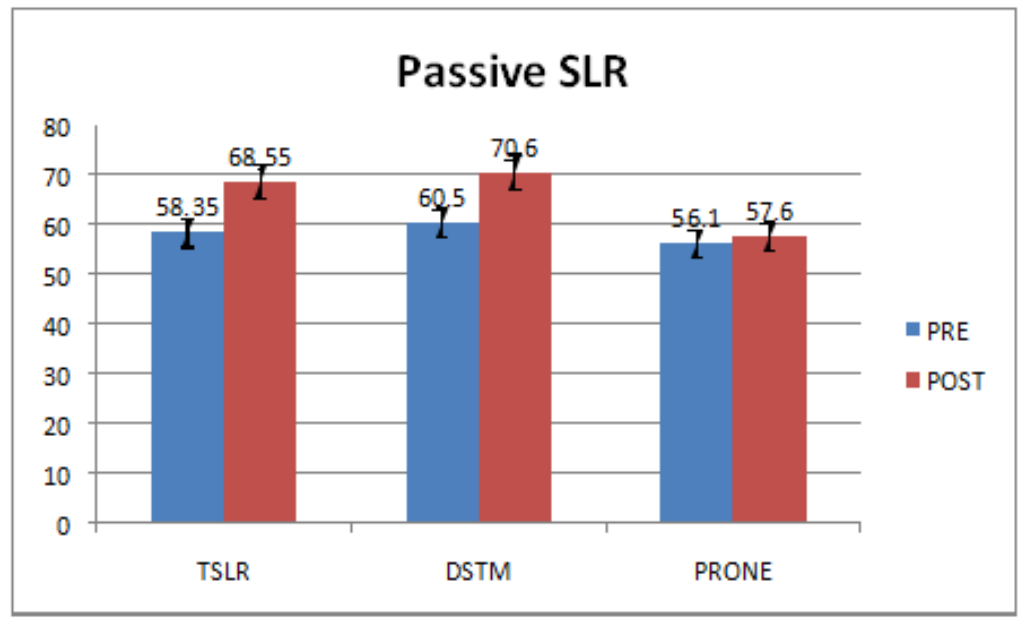

Graph -2

The graph -2 illustrates that there was improvement in passive range of motion in groups receiving mulligan traction straight leg raise (gr-1) and dynamic soft tissue mobilization (gr-2), but not in the control group (gr-3) from pre intervention measurement to post intervention measurement for a period of 3 weeks. Result showing that there was main effects for time i.e., $f(1,28,0.05)=1.115 \mathrm{E} 3, \mathrm{p}=0.000$ and main effects for groups $\mathrm{F}(1,28,0.05)=25.090, \mathrm{p}=0.0000$ main effects qualified into Time $\times$ Group interaction i.e. $\mathrm{F}(1,28$, $0.05)=175.527, \mathrm{p}=0.000$. Turkey' post Hoc analysis shows that there was improvement for all the three groups improved significantly with time although the control group only improved (1.5 degree) at the end of 3 weeks which is not clinically significant. At the end of 3 wks all the 3 groups were significantly difference from each other but the difference between group-1 and group-2 was not clinically significant (2.05 degree).

\section{Discussion}

The study implied Mulligan's traction straight leg raise and dynamic soft tissue mobilization improved active range of knee and passive straight leg raise range of motion in normal individuals. The improvement of control group was not clinically significant. In this study both active and passive range had improved in both the experimental groups. Passive range of motion (ROM) was improved as the muscle was being elongated beyond the slack length of the tissue and active range was improved as the muscles could lengthen in the outer range gained by the stretching technique.

Mulligan's Traction SLR had improved the hamstrings flexibility in experimental group (gr-A) This could be due to following mechanism. Changed viscoelastic property that occurred with "creep," where by the tension in the muscle-tendon unit diminished over time. The hamstrings muscle responded to the viscoelastic change to the tensile loads. With repetitive stretching there was little alteration of muscle-tendon unit implied that, a minimum number of stretches would lead to most of the elongation. Also greater peak tensions and greater energy absorption occurred at faster stretch rates, suggesting that, the risk of injury in a stretching regimen might be related to the stretch rate, and not to actual technique. All of these important clinically considerations could be related to the viscoelastic characteristics of muscletendon unit.

Another factor that might attribute to the lengthening of the hamstrings muscle during TSLR might be due to the inhibition of the hamstrings muscles itself. In this technique, various receptors exerted an inhibitory influence on lower limb alpha motor neuron activity. Golgi tendon organs around the knee, hip and spine probably initiated various segmental reflex pathways during traction of the limb. Likewise, Golgi tendon organs were activated during large amplitude stretching movements such as SLR. This processing of information in the nervous system might inhibit the activity of the muscles being lengthened during SLR by dampening the afferent activity of type II muscle spindles or by decreasing motor neuron excitability via 1-b fibers. Hence, improvement in range of SLR might be directly related to inhibition of the hamstrings muscles.

The nerve tissue might be a possible limiting factor in range of motion and it was suggested that the increase in range of motion (with no corresponding change in length of torque) by stretch exercise, could result from mobilization of a nervous tissue. The improvement with neural mobilization might be explained for the fact that the nerve tissue was stressed at the extreme of joint position (i.e. in a direction of movement produced by tight muscle), wherein the muscle and fascia were also maximally elongated. When neural tissues, which passed through the tight muscle, were mobilized, the restriction to mobility faced at interface of nerve and 
muscle were overcome and the flexibility of nerve-muscle unit had been increased. More the nerve was manipulated, more was the movement at the mechanical interface and more was the flexibility (excursion).

Along with the hamstrings, the deep fascia of the lower limb and the soft tissues of the pelvis, including neural tissue could limit a straight leg raise test. In the same way, these non-contractile tissues could come under tension during passive or active movements of hip flexion or knee extension. If tension of non-contractile tissue limited indirect measures of hamstrings flexibility, i.e., straight leg raise or active knee extension tests, then use of a stretching technique that emphasizes these tissues, along with the hamstrings. The present study was supported by the following studies.

Pratishtha, et al. (2012) Conducted study on Effect of Mulligan Stretching Techniques (TSLR and BLR) on Biceps Femoris Muscle and Pelvic Rotation by Using Surface EMG and Bubble Inclinometer respectively, concluded that Mulligan TSLR stretch was more effective than BLR stretch in improving biceps femoris muscle performance, flexibility and pelvic rotation as in the TSLR group the EMG activity decreased by $22.52 \%$. [27]

Laessoe U, Paterson PM studied on stretch tolerance on 10 healthy individuals. The aim of this study was to investigate the effects of these two techniques on knee range of motion of normal subjects (30 females [mean age $21.4+/-1.2$ years, range 1924]) in the slump position. Knee flexion angle of the right leg was measured using a universal $360^{\circ}$ goniometer while in a fully slumped position, prior to and after the application of the respective mobilization technique. The tensioner technique brought about a significant decrease in knee flexion angle $(\mathrm{P}=0.003)$ with a mean percentage change of $14.7+/-11.8 \%\left(3.4+/-2.5^{\circ}\right)$. The slider technique brought about a significant decrease in knee flexion angle $(\mathrm{P}<0.001)$ with a mean percentage change of $19.9+/-15 \%\left(4.3+/-2.6^{\circ}\right)$. There was a non-significant between-group difference for the effect of the two techniques on range of motion $(\mathrm{P}=0.075)$. The findings of this study indicate that in normal female subjects, both the tensioner and slider techniques have a positive and significant effect on improving knee extension range of motion in the slump position. This could decrease the sensitivity of the sciatic nerve and the neuromeningeal structures to mechanical load. [28]

Jo M. Fasen, Annie M. O'connor studied that the addition of a neuromobilization component may be beneficial to hip flexion and knee extension range of motion. The improvement seen in the neuromobilization group emphasizes the fact that flexibility is influenced not only by muscle elasticity but also by connective tissue/nervous tissue extensibility. Stretching of muscle applies tension to other structures such as the joint capsule and fascia, which are made up of different tissue than muscle with different passive resistance to stretch with the addition of neural tension during passive hamstrings stretch despite no change in the EMG response indicates that passive extensibility of neural tissues can limit hamstrings flexibility. One hundred subjects between the ages of 21 and 57 were enrolled in the study. All 100 subjects were included in a randomized controlled trial of 5 different groups comparing different hamstrings-stretching techniques. Outcome measures, including hamstrings length and perceived level of hamstrings tightness, were recorded on all subjects initially, at 4 weeks, and at 8 weeks. After 4 weeks of stretching, there was a statistically significant improvement in hamstrings length $(\mathrm{p}, 0.05)$ using active stretches as compared with passive stretches. From weeks 4 through 8 , hamstrings length for the active stretching groups decreased. After 8 weeks of stretching, the straight leg raise (SLR) passive stretch group had the greatest improvement in hamstrings length. Improvement in hamstrings flexibility was greatest for the SLR passive stretch. [29]

Hall, et al. (2006) conducted a pilot study on Mulligan Traction Straight Leg Raise (TSLR) to investigate the range of motion in patient with low back pain. Aim of the study was to determine the immediate effects of Mulligan Traction Straight Leg Raise (TSLR) technique on range of the SLR test. They concluded that there was a significant increase in range of SLR by 11 degree in experimental group. [30] Consistent with the findings of above study, in our study, the improvement of range was 10.2 degree.

Antonio Cacho, McNee Catherine, James Riches, James Walsh (2001) conducted an experimental, pre-test, intervention, post-test design study. The Mulligan traction straight leg raise (SLR) technique is used therapeutically to increase range of SLR when it is limited due to low-back dysfunction or hamstrings tightness. The effects of this technique were investigated in 26 normal subjects (mean age 26 years; 13 male). As the movement of SLR comprises hip flexion and posterior pelvic rotation were measured pre- and post-intervention using two bubble inclinometers. Following the intervention, the mean range of SLR significantly increased by 2.7 degree. Hip flexion was the measure reason for increased range of SLR following the intervention, indicating an increase in hamstring muscle stretch tolerance. [31]

Ganer et al (2014) investigated the effect of Posterior to Anterior mobilization and Traction SLR on pain and neuro-dynamic mobility in patients of low back pain. They found Traction SLR and posterior to 
anterior mobilization are effective in improving ROM and pain in patients suffering from low back pain. However there was no statistically significant difference between Traction SLR and posterior to anterior mobilization in improving Pain and ROM in patients suffering from low back pain. [32] Toby Hall et al conducted a study to determine the immediate effects of the Mulligan traction straight leg raise technique. This study concluded that traction SLR is effective there was significant increase in the range of SLR by $11^{\circ}$ in subject. [31]

Jesudas and Shriwas (2014) investigated the effect of Mulligan Traction Straight Leg Raise and Muscle Energy Technique on hamstrings tightness in asymptomatic males. They recruited 60 subjects $(n=60)$ with hamstrings tightness randomly divided into two groups. Each group consist of 30 male subjects. However group A had 28 as two drop out and group B had 26 as four dropout .Group A was treated with Mulligan TSLR and Group B had MET. The total intervention session was 3 times a week on alternate days for three consecutive weeks. The outcome measures were measured by active knee extension ROM by universal goniometer. The Mean and SD of knee Range Motion at 0 week for Group A was found to be 37.19 and 7.38 respectively where as that of Group B to be 39.29 and 7.03 respectively. When a comparison of the mean value for knee ROM at Median (0 week - week 1) was done between Group A and Group B, the $t$-value was found to be 1.989 with $\mathrm{P}>0.05$. It means that there was no significant difference in improvement of knee ROM when compared between Group A and Group B at Median in the 1st week. When a comparison of the mean for knee ROM for the 2nd week was done by comparing it with the Mean of the 0 week of both the Groups A and B. It shows that there was a significant improvement of Knee ROM in both the Group A and B. When the Mean value for Knee ROM was compared at Median (0 week - 2 weeks) between Group A and Group B, t value was found to be 1.316 with $\mathrm{P}>0.05$. [33] Thus, in this study, hip flexion and posterior pelvic rotation, the repetitive hamstrings muscle stretch along with mobilisation of sciatic nerve, stretching of posterior capsule of hip, deep fascia and neuro-meningeal structures during the Mulligan Traction Straight Leg Raise Technique might improve the range of SLR.

In the dynamic STM, the hamstrings muscle group received progressive dynamic techniques that worked in synchrony as the muscle moved to the end ROM. The final technique eccentrically worked the muscle at its functional length with the result that hamstrings flexibility was optimized. DSTM had improved range of motion (Group-B) because of following mechanisms: In dynamic soft tissue mobilization, incorporating active contraction into a massage protocol might increased muscle perfusion and decreased muscle stiffness. DSTM induced improve metabolic process in muscle cells which in turn caused an increased in temperature that led to decreased muscle viscosity and allowed for a smoother contraction. Muscles were more pliable and accommodating to forces placed on the muscle leading to increased flexibility. DSTM might have improved the mobility by releasing trigger points and loosen adhesion in connective tissue that could bind muscles. DSTM involves physiological mechanisms like autogenic inhibition via recruitment of the Golgi tendon organs and reciprocal inhibition which caused inhibition of the target muscle following the contraction of the opposing muscle. This stretching leads to relaxation or inhibition of the stretched muscle and thus led to increased hamstrings flexibility. The present study was supported by the following studies.

D Hopper et al (2005) conducted a study to investigate the effect of dynamic soft tissue mobilisation on hamstrings flexibility in healthy male subjects. They compared control group, classic soft tissue mobilisation group and dynamic soft tissue mobilisation group. In their study they demonstrated that a significant increase in hamstrings length could be achieved by identifying are of hamstrings tightness and targeting intervention to this area using dynamic therapy. They concluded that, increase in hamstrings flexibility was significantly greater in the dynamic STM group than either the control or classic STM groups with mean (standard deviation) increase in degrees in measures of 4.7 (4.8), 20.04 (4.8), and 1.3 (3.8), respectively.[25]

Diana Hopper et al (2005) on the evaluation of the effect of two massage techniques on hamstrings muscle length in competitive female hockey players. They compared the classic massage with dynamic soft tissue mobilisation and concluded that both these standardized classic massage and dynamic soft tissue mobilisation interventions have an immediate effect on improving hamstrings length. The AKE test demonstrated a significant improvement in hamstrings length following massage in both groups $(F=7.66$, $p=0.01)$. This increase was comparable between the two massage groups $(F=0.164, p=0.69)$. Post-hoc linear contrast showed no maintenance over $24 \mathrm{~h}$ in either group, (classic $F(1,18)=2.106, p=0.164$, DSTM $F(1,15)=0.599, p=0.451)$. Passive KE showed that both classic massage and DSTM had a significant effect on hamstrings length in competitive female field hockey players. [34] 
K. Kotteeswaran, Josyula Snigdha and Alagesan (2014), investigated the effect of proprioceptive neuromuscular facilitation stretching and dynamic soft tissue mobilization on hamstrings flexibility 103 subjects satisfying selection criteria in the age group of 20-35 years were randomly allotted in to PNF or DTSM group and were assessed for hamstrings flexibility and pain using active knee extension (AKE) test. The post intervention mean \pm SD value of AKE test in PNF CRAC group is $20.38 \pm 4.23$ and DSTM group is $18.10 \pm$ 4.55. The post intervention mean \pm SD value of NPRS in PNFCRAC group is $4.41 \pm 0.95$ and DSTM group is $2.31 \pm 1.04$. The independent $t$ test for between group analysis after intervention for AKE test and NPRS shows significant difference between groups with $\mathrm{P}<0.0001$. DSTM is more effective than PNFCRAC in increasing AKE. This study concludes that dynamic soft tissue mobilization is more effective than proprioceptive neuromuscular facilitation contract relax antagonist contract stretching in improving hamstrings flexibility. [35]In this study, the increased range of motion may be because of dynamic soft tissue mobilisation in specific structured technique in which a therapist identifies a target area of muscle tightness and focuses the intervention in that particular area. This study shows that, dynamic soft tissue mobilisation also had an effect on hamstrings length and hence on flexibility. Also research studies have shown that, a 20 minute classical massage has no effect. So, it may be worthy of clinical consideration.

Youdas JW et al (2005) conducted a study to examine the factors of gender and age, stratified by 10year increments, on hamstrings muscle length (HML) as measured by passive straight-leg raise (PSLR) and popliteal angle (PA) differences in hamstrings muscle length between men and women were examined for a large group of healthy adults over a wide range of ages. Females demonstrated greater flexibility than their male counterparts. HML differed significantly (P.001) between genders for both methods of measurement, with females demonstrating greater flexibility than their male counterparts. The difference between genders was $8^{\circ}$ for PSLR and $11^{\circ}$ for PA. [36] In this study only male subjects were taken as females show greater hamstrings muscle flexibility than males.

\section{Conclusion}

The result of the study suggested that all the intervention given in the present study i.e., Mulligan traction straight leg raise and dynamic soft tissue mobilization are equally effective to improve hamstrings flexibility in asymptomatic male subjects Therefore after analyzing the data the following conclusions were drawn:

1) There was no significant difference in between Mulligan traction straight leg raise and Dynamic Soft Tissue Mobilization in the flexibility of the hamstrings muscles.

2) Mulligan traction straight leg raise and Dynamic Soft Tissue Mobilization were equally effective in improving the flexibility of hamstrings muscles.

\section{Limitation}

1) As this study was limited to the effect of stretching on the hamstrings muscle, other studies are needed to evaluate the effect of stretching on other muscle groups such as gastrocnemius, soleus and iliotibial band.

2) The result of this study could not be generalized to all population of having hamstrings tightness.

3) Further research examining the effects of stretching on individual in other age groups would be of interest.

4) Female subjects were not taken in this study. Further study on females can be done or a Comparative study between genders would be of interest.

5) Studies with longer duration and larger sample size were recommended with longer follow-up period to assess long term benefits

\section{References}

[1]. Chandler T, Kibler J, UHL WB, Stone E (1990) Flexibility comparisons of junior elite tennis players to other athletics. American Journal Sports Medicine 18(2): 134-136.

[2]. Campanelli LC (1996) Mobility changes in older adults: Implications for practitioners. Journal of Aging and Physical Activity 4(2): $115-118$.

[3]. Wang SS, Whitney SL, Burdett RG and Janosky JE (1993) Lower extremity muscular activity in long distance runners. Journal of Orthopedics \& Sports Physical Therapy 17(2): 102 -107.

[4]. American college of sports medicine, (ACSM) Guidelines for exercise testing and prescription (2000) 6: 158-162.

[5]. Bandy WD, Iron JM, Briggler M (1998) The effect of static stretch and dynamic range of motion training on the flexibility of the hamstrings muscles. Journal of Orthopedics Physical Therapy 27(4): 295-300.

[6]. MJ (1988) Science of stretching, Champaign, III Ed. Human Kinetics Book.

[7]. www.soccer-training-info.com

[8]. Feland JB, Myrer JW, Fellingham GW (2001) The Effect of duration of stretching of the hamstrings muscle group for increasing range of motion in people aged 65 years or older. Physical therapy. 81(5): 1110-1117.

[9]. Www.fitnessmanagement.com

[10]. Turner D, Gossman RM, Nicholson CG and Lemons J (1988) Comparison of cyclic and sustained passive stretching using a mechanical device to increase resting length of hamstrings muscles. Phys Ther 69(3): 314-320.

[11]. Odunaiya NA, Hamzat TK, Ajayi OF (2005) The Effects of Static Stretch Duration on the Flexibility of Hamstrings Muscles. African Journal of Biomedical Research 8; $79-82$. 
[12]. Erukula G, Demirkan F, Aiper B (2002) Hamstrings shortening in healthy Adults. Journal of Back and Musculoskeletal Rehabilitation 16 (2):77-81.

[13]. James M, Kolt G, McConville J, Bate P (1998) The effects of a Feldenkrais Program and relaxation procedures on hamstrings length. Australian Journal of Physiotherapy 44(1): 50-54.

[14]. Decoster LC, Scanlon RL, Horn KD, Cleland J (2004) Standing and supine hamstrings stretching are equally effective. Journal of Athletic Training 39(4): 330-334.

[15]. Williford HN et al (1986) Evaluation of warm up for improvement in flexibility. American Journal of Sports Medicine 14(4): $316-$ 9.

[16]. Sady SP, Wortman M, Blanke D (1982) Flexibility training ballistic, static and proprioceptive neuromuscular facilitation. Arch Physical Med. Rehabilitation 63(6): 261-3.

[17]. Spernoga SG, Uhl TL et al (2001) Duration of Maintained Hamstrings Flexibility after One-Time, Modified Hold Relax Stretching Protocol. Journal of Athletic Training 36(1): 44-48.

[18]. Best TM (1995) Clinical Sports medicine 14(3):669-686.

[19]. Kisner C, Calby LA. The Therapeutic exercise, Foundations and techniques. III Ed.

[20]. Witvrow E, Danneels L et al (2003) American Journal of Sports Medicine 31 (1): 41-46.

[21]. Taylor DC, Dalton JD et al (1990) Viscoelasticity properties of muscle - tendon units. American Journal of Sports Medicine 18(3): 300-309.

[22]. Back BK et al (1985) A comparison of muscular tightness in runners and non runners and the relation of muscular tightness to low back pain in runners. J Orthop Sports Phys Ther. 6: 315-323.

[23]. Safran MR, Garrett WE, Seaber AV et al (1988) The role of warm up exercise is muscular injury prevention. American Journal of Sports Medicine 16: 123-129.

[24]. Hartig DE, Henderson JM (1999) Increasing hamstrings flexibility decreases lower extremity overuse injuries in military basic trainees. American Journal of Sports Medicine 27(2): 173-6.

[25]. Hopper D, Deacon S et al (2005) Dynamic soft tissue mobilization increases hamstrings flexibility in healthy male subjects. British Journal of Sports Medicine 39: 594-598.

[26]. Nelson RT and Bandy WD (2004) Eccentric Training and Static Stretching Improve Hamstrings Flexibility of High School Males. Journal of Athletic Training 39(3): 254-258.

[27]. Pratishtha K, Jagga V (2012) Effect of Mulligan Stretching Techniques (TSLR AND BLR) on Biceps Femoris Muscle and Pelvic Rotation by Using Surface EMG and Bubble Inclinometer Respectively. Journal of Exercise Science and Physiotherapy 8(1): 3942.

[28]. Laessoe U, Patterson PM et al (1980) Stretching; effect of passive muscle stiffness of short hamstrings. Arch Phys Med Rehabilitation 77(7): 688-692.

[29]. Fasen JM, O'Connor AM et al (2009) A randomized controlled trial of hamstrings stretching: comparison of four techniques. Journal of Strength and Conditioning Research 23(2): 660-667. www.nsca-jscr.org.

[30]. Hall T, Beryerlein C, Hansson U, Huntech L, Sainburg D (2006) Mulligan traction straight leg raise; A pilot study to investigate effect on range of motion in patient with low back pain. Journal of manual and manipulative therapy 14(2): $95-100$.

[31]. Hall T, Hardt S, Schafer A, Wallin L (2006) Mulligan bent leg raise technique-a preliminary randomized trial of immediate effects after a single intervention Man Ther. 11(2): 130-5.

[32]. Naveen G et al (2014) Comparison between posterior to anterior mobilization and traction slr on pain and neurodynamic mobility in patients of low back pain. International Journal of Physiotherapy and Research, Int J Physiother Res 2(1): 383-87. ISSN 2321-1822

[33]. Jesudas, Shriwas (2014) A comparison between mulligan traction straight legraise technique vs muscle energy technique on hamstrings tightness in asymptomatic male. International Journal of Physiotherapy and Research, Int J Physiother Res 2(2): $412-17$. ISSN 2321-1822

[34]. Hopper D, Conneely M, Chromiak F, Canini E (2005) Evaluation of the effect of two massage techniques on hamstrings muscle length in competitive female hockey players, Physical Therapy in Sports 6137145.

[35]. Kotteeswaran K, Snigdha J, Alagesan J (2014) Effect of proprioceptive neuromuscular facilitation stretching and dynamic soft tissue mobilization on hamstrings flexibility in subjects with low back ache - single blinded randomised controlled study. International Journal of Pharma and Bio Sciences, Int J Pharm Bio Sci 5(3): (B) 228 - 233, ISSN 0975-6299.

[36]. Youdas JW, Krause DA, Hollman JH, Harmsen WS, Laskowski E (2005) The influence of gender and age on hamstrings muscle length in healthy adults. J Orthopedics Sports Phys Ther. 35(6): 388. 